\title{
ON.THE EXPLICITE DEFINING RELATIONS OF ABELIAN SCHEMES OF LEVEL THREE
}

\author{
HISASI MORIKAWA
}

Dedicated to the memory of Professor TADAsi Naxayama

It is known classically that abelian varieties of dimension one over the field of complex numbers may be expressed by non-singular Hesse's canonical cubic plane curves, $X_{0}^{3}+X_{1}^{3}+X_{-1}^{3}-6 \gamma X_{0} X_{1} X_{-1}=0$. The purpose of the present paper is to generalize this idea to higher dimensional case.

Let $\mathbf{Z}(3)$ be the residue group of the additive group $\mathbf{Z}$ of integers modulo $3 \mathbf{Z}$ and $\mathbf{Z}(3)^{r}$ be the $r$-times direct sum of $\mathbf{Z}(3)$. We mean by $\mathbf{Z}(3)^{+r}$ the subset of $\mathbf{Z}(3)^{r}$ consisting of all the elements $\left(a_{1}^{+}, \ldots, a_{r}^{+}\right)$such that $a_{i}^{+}=0$ or 1 $(1 \leq \boldsymbol{i} \leq \boldsymbol{r})$. Then, roughly speaking, our result may be expressed as follows: a generic abelian variety with a positive divisor $U$ such that $l(U)=1^{1)}$ is defined by relations of the following type

$$
\begin{aligned}
& \Delta_{2} Y_{a+b} Y_{-a+b} Y_{b}-\sum_{c \in Z(3)} r a, c Y_{c+b}^{3}=0 \\
& \Delta_{1} Y_{a+b} Y_{-a+b}-\sum_{c+\in Z(3)+r} \beta_{a, c^{+}} Y_{c^{++b}} Y_{-c^{++b}}=0, \quad\left(a, b \in \mathbf{Z}(3)^{r}\right) .
\end{aligned}
$$

\section{$\S 1$. Formal theta functions of level $n$ and the scheme $A(r, n)$ associated with them}

1.1. We mean by $\mathbf{Z}$ and $\mathbf{Q}$ the ring of intergers and the field of rational numbers. We mean by $\mathbf{Z}^{r}$ the $r$-times direct sum of the $\mathbf{Z}$-module $\mathbf{Z}$ and by $\mathbf{Q}^{r}$ the $r$-times direct sum of the Q-module Q. Let $\{W(i ; \alpha), W(j, l ; \beta) \mid 1 \leq i, j, l \leq r$; $\alpha, \beta \in \mathbf{Q}\}$ be a system of indeterminates on which rational numbers operate such that $W(i ; \alpha)^{\gamma}=W(i ; \alpha \gamma), W(j, l ; \beta)^{\gamma}=W(j, l ; \beta \gamma)$. We denote by $I$ the ideal in the polynomial ring $\mathrm{Z}[\{W(i ; \alpha), W(f, l ; \beta)\}]$ generated by

$$
\begin{array}{r}
W(i ; 0)-1, W(j, l ; 0)-1, W(i ; n \boldsymbol{\alpha})-W(i, \overbrace{\alpha) \cdots W(i ; \alpha),}^{n}, \\
W(j, l ; n \beta)-W(j, l, \beta \overbrace{\cdots W(j, l}^{n} ; \beta)
\end{array}
$$

Received May 10, 1965.

1) $l(U)$ means the rank of the module of the multiples of $-U$. 


$$
\begin{aligned}
& W(i ; \alpha) W(i ; \beta)-W(i ; \alpha+\beta), W(j, l ; \alpha) W(j, l ; \beta)-W(i, l ; \alpha+\beta), \\
& W(i, l ; \alpha)-W(l, j ; \alpha),
\end{aligned}
$$

$$
(1 \leq i, j, l \leq r ;, \alpha, \beta \in \mathbf{Q}, \boldsymbol{n}=1,2, \ldots)
$$

We mean by $U_{j}^{\alpha}$ and $Q_{j l}^{\beta}$ the images of $W(i ; \alpha)$ and $W(j, l ; \beta)$ in the residue ring $B=\mathbf{Z}[\{W(i ; \alpha), W(j, l ; \beta)\}] / I$. Then it follows the relations :

$$
\begin{aligned}
& U_{i}^{n \alpha}=\overbrace{U_{i}^{\alpha} \cdots \cdot U_{i}^{\alpha}}^{n}, Q_{i l}^{n \beta}=\overbrace{Q_{i l}^{\beta} \cdots Q_{i l \tau}^{\beta}}^{n} U_{i}^{0}=1, Q_{l i}^{0}=1, \\
& U_{i}^{\alpha} U_{i}^{\beta}=U_{i}^{\alpha+\beta}, Q_{l j}^{\alpha} Q_{l j}^{\beta}=Q_{j l}^{\alpha+\beta} \\
& \left(U_{i}^{\alpha}\right)^{\gamma}=U_{i}^{\alpha \gamma}, \quad\left(Q_{l j}^{\beta}\right)^{\gamma}=Q_{j l}^{\beta \gamma}, \\
& Q_{j l}^{\alpha}=Q_{j l}^{\alpha},
\end{aligned}
$$

$$
(1 \leq i, j, l, \leq r ; \alpha, \beta, \gamma \in \mathbf{Q} ; \boldsymbol{n}=1,2,3, \ldots) .
$$

We shall use the following brief notations:

$$
\begin{aligned}
& U(\alpha)=\prod_{i=1}^{r} U_{i}^{\alpha_{i}}, \\
& Q(\alpha, \beta)=\prod_{i, j=1}^{r} Q_{i j}^{\alpha_{i j} \beta_{j}}, \\
& \left(\alpha=\left(\alpha_{1}, \ldots, \alpha_{r}\right), \beta=\left(\beta_{1}, \ldots, \beta_{r}\right) \in \mathbf{Q}^{r}\right) .
\end{aligned}
$$

Then if follows

$$
\begin{aligned}
& U(\alpha) U(\beta)=U(\alpha+\beta), \\
& Q(\alpha+\beta, \gamma+\delta)=Q(\alpha, \gamma) Q(\alpha, \delta) Q(\beta, \gamma) Q(\beta, \delta), \\
& \quad\left(\alpha, \beta, \gamma, \delta \in \mathbf{Q}^{r}\right) .
\end{aligned}
$$

1.2. We mean by Hom $\left(\mathbf{Z}^{r}, G_{m}\right)$ the functor of the category of commutative rings into the category of ablian groups such that Hom $\left(\mathbf{Z}^{r}, G_{m}\right)(A)$ means the group of all the homomorphisms of the additive group $\mathbf{Z}^{r}$ into the multiplicative group of the units in $A$. By virtue of (7) $U$ may be considered as an element in Hom $\left(\mathbf{Z}^{r}, G_{m}\right)(B)$. For each $\alpha$ in $\mathbf{Q}^{r}$ we may construct an element $Q(\alpha)$ in Hom $\left(\mathbf{Z}^{r}, G_{m}\right)(B)$ given by

$$
Q(\alpha)(m)=Q(\alpha, m),\left(m \in \mathbf{Z}^{r}\right)^{2} .
$$

We mean by $Q(\alpha) U$ the product of $Q(\alpha)$ and $U$ in Hom $\left(\mathbf{Z}^{r}, G_{m}\right)$.

Definition 1. We mean by a formal rational power series in $Q$ of restricted

2) We change the notation $Q(\alpha)$ slightly. In [1] and [2] we defined $Q(\alpha)$ by $Q(\alpha)(m)$ $=Q(\alpha, m)^{2}$. 
type a formal rational power series $\sum_{\left(\alpha_{i j}\right)} \lambda_{\left(\alpha_{i j}\right)} \prod_{i \leq j} Q_{i j}{ }^{\alpha_{i j}}$ satisfying the conditions: $1^{\circ}$ there exists a positive integer $m$ such that $m \alpha_{i j} \in \mathbf{Z}(1 \leq i, j \leq r)$ for $\left(\alpha_{i j}\right)$ satisfying $\lambda_{\left(\alpha_{i j}\right)} \neq 0,2^{\circ}$ for any positive integer $n$ there exist only a finite number of terms $\lambda_{(\alpha i j)} \prod_{i \leq j} Q_{i j}{ }^{\alpha_{i j}}$ such that $\lambda_{\left(\alpha_{i j}\right)} \neq 0$ and $\alpha_{i i} \leq n(1 \leq i \leq r)$.

All the formal rational power series in $Q$ of restricted type with coefficients in $\mathbf{Z}$ form a commutative integral domain. We denote it by $\mathbf{Z}[[Q]]$.

We shall now give the definition of formal theta functions:

Definition 2. We mean by a formal theta functions of level $n$ with coefficients in $\mathrm{Z}[[Q]]$ a formal power series in $U_{i}^{2}, U_{i}^{-2}(1 \leq i \leq \boldsymbol{r})$

$$
\varphi(U)=\sum_{m \in \mathbb{Z}^{r}} \lambda_{m} U(m)^{2}
$$

with coefficients $\lambda_{m}$ in $Z[[Q]]$ such that

$$
\varphi(Q(m) U)=Q(m, m)^{-n} U(m)^{-2 n} \varphi(U),\left(m \in \mathbf{Z}^{r}\right) .
$$

1.2. We denote by $\mathbf{Z}(n)$ the residue group of $\mathbf{Z}$ modulo $n \mathbf{Z}$. We denote by $0,1,2, \ldots, n-1$ sometimes the elments in $\mathbf{Z}(n)$ and sometimes integers $0,1,2, \ldots, n-1$ in $\mathbf{Z}$ so that $a / n(a \in \mathbf{Z}(n))$ makes sense. We denote by $\mathbf{Z}(n)^{r}$ the $r$-times direct sum of $\mathbf{Z}(\boldsymbol{n})$. By virtue of the difference equation (10) the coefficients $\lambda_{m}$ of a formal theta function $\sum_{m \in \mathbf{Z}^{r}} \lambda_{m} U(m)^{2}$ of level $n$ are given by

$$
\begin{aligned}
\lambda_{n m+g}=\lambda_{g} Q\left(\frac{g}{n}, \frac{g}{n}\right)^{-n} Q\left(m+\frac{g}{n}, m+\frac{g}{n}\right)^{n}, & \\
& \left(g \in \mathbf{Z}(n)^{r}, m \in \mathbf{Z}^{r}\right) .
\end{aligned}
$$

We shall introduce the canonical system of formal theta functions of level $n$ :

$$
\begin{aligned}
X_{n, g}(Q \mid U)=\sum_{m \in \mathbf{Z}^{r}} Q\left(m+\frac{g}{n}, m+\frac{g}{n}\right)^{n} U\left(m+\frac{g}{n}\right)^{2 n}, & \\
& \left(g \in \mathbf{Z}(n)^{r}\right) .
\end{aligned}
$$

Then it follows the formulae

$$
\begin{aligned}
& X_{n, g}\left(Q \mid U^{-1}\right)=X_{n,-g}(Q \mid U) \\
& X_{n, g}\left(Q \mid Q\left(\frac{h}{n}\right) U\right)=Q\left(\frac{h}{n} \cdot \frac{h}{n}\right)^{-n} U\left(\frac{h}{n}\right)^{-2 n} X_{n, g+h}(Q \mid U), \\
& \quad\left(g, h \in \mathbf{Z}(n)^{r}\right) .
\end{aligned}
$$

From (11) it follows that a formal theta function $\sum_{m \in \mathbf{Z}^{r}} \lambda_{m} U(m)^{2}$ is a linear combination of $X_{n, g}(Q \mid U)\left(g \in \mathbf{Z}(n)^{r}\right)$ as follows : 


$$
\sum_{m \in Z^{r}} \lambda_{m} U(m)^{2}=\sum_{o \in Z(n)^{r}} \lambda_{g} Q\left(\frac{g}{n} \cdot \frac{g}{n}\right)^{-n} X_{n, g}(Q \mid U) .
$$

Putting $U=1$, we have a system of elements in $Z[[Q]]$ :

$$
T_{n, g}(Q)=X_{n, g}(Q \mid 1),\left(g \in \mathbf{Z}(n)^{r}\right),
$$

From (13) it follows

$$
T_{n,-g}(Q)=T_{n, g}(Q),\left(g \in \mathbf{Z}(n)^{r}\right) .
$$

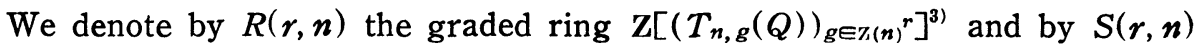
the projective scheme Proj $(R(r, n))$ of the graded ring $R(r, n)$, where deg $T_{n, g}(Q)=n\left(g \in \mathbf{Z}(n)^{r}\right)$. Since $R(r, n)$ is a commutative noetherian integral domain, the scheme $S(r, n)$ is irreducible, reduced and noetherian.

By virtue of (12) and (15) it follows the fundamental property of theta functions of level $n$ :

Proposition 1. The formal theta functions of level $n X_{n, g}(Q \mid U)\left(g \in \mathbf{Z}(n)^{r}\right)$ form a base of the formal theta functions of level $n$ over any field containing $R(r, n)$.

We denote by $O_{S(r, n)}$ the structure sheaf of $S(r, n)$ and by $O_{S(r, n)}\left[\left(X_{n, g}(\mathrm{Q} \mid\right.\right.$

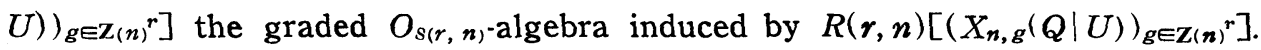
We mean by $A(r, n)$ the projective $S(r, n)$-scheme $\operatorname{Proj}_{s(r, n)}\left(O_{S(r, n)}\left[\left(X_{n, g}(Q)\right.\right.\right.$

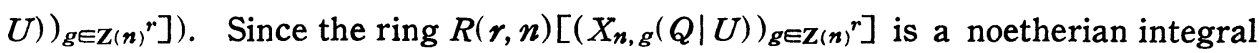
domain, $A(r, n)$ is also a noetherian irreducible reduced scheme.

\section{$\S 2$. Formal theta functions of level three}

2.1. We shall use the following notations;

$\mathbf{Z}(3)$ : the residue group of $\mathbf{Z}$ modulo $3 \mathbf{Z}$, we denote by $0,1,-1$, sometimes the elements in $\mathrm{Z}(3)$ and sometimes integers $0,1,-1$ in $\mathrm{Z}$ so that $a / 3(a \in(3))$ makes sense,

$\mathbf{Z}(3)^{+}$: the subset $\{0,1\}$ in $\mathbf{Z}(3)$,

$\mathbf{Z}(3)^{r}$ : the r-times direct sum,

$\mathbf{Z}(3)^{+r}$ : the subset in $\mathbf{Z}(3)^{r}$ consisting of all the elements $\left(a_{1}^{+}, \ldots, a_{r}^{+}\right)$ such that $a_{i}^{+}=0$ or $1,(1 \leq i \leq r)$,

3) Since $T_{n}, g\left(g \in Z(n)^{r}\right)$ are modular forms of degree $r$ for certain congruence subgroup, $Z\left[T_{n}, g(Q)\right)_{\left.y \in Z(n)^{r}\right]}$ forms a graded ring. 
$a, b, c, \ldots:$ the elements in $\mathrm{Z}(3)^{r}$,

$a^{+}, b^{+}, c^{+}, \ldots:$ the elements in $\mathbf{Z}(3)^{+r}$,

$$
T_{a}=T_{3}, a(Q)=\sum_{m \in \mathbf{Z}^{r}} Q\left(m+\frac{a}{3}, m+\frac{a}{3}\right)^{3} \quad\left(a \in \mathbf{Z}(3)^{r}\right),
$$

$\left(T_{a^{+}+b^{+}} T_{-a^{+}+b^{+}}\right):$the $2^{r} \times 2^{r}$-matrix of which $\left(a^{+}, b^{+}\right)$-component is

$$
T_{a^{++} b^{+}} T_{-a^{++} b^{+}}
$$

$\left(T_{a+b} T_{-a+b}\right):$ the $3^{r} \times 2^{r}$-matrix of which $\left(a, b^{+}\right)$-component is

$$
T_{a+b^{+}} T_{-a+b^{+}}
$$

$\left(T_{a+b} T_{-a+b} T_{b}\right):$ the $3^{r} \times 3^{r}$-matrix of which $(a, b)$-component is

$$
T_{a+b} T_{-a+b} T_{b},
$$

$\left(T_{a+b}^{3}\right):$ the $3^{r} \times 3^{r}$-matrix of which $(a, b)$-component is $T_{a+b}^{3}$,

$$
\begin{aligned}
& \Delta_{1}(T)=\operatorname{det}\left(T_{a^{+}+b^{+}} T_{-a^{+}+b^{+}}\right), \\
& \Delta_{2}(T)=\operatorname{det}\left(T_{a+b}^{3}\right) \text {, } \\
& \left.\alpha_{a^{+}, b^{+}}(T)\right)=\Delta_{1}(T)\left(T_{a^{+}+b^{+}} T_{-a^{+}+b^{+}}\right)^{-1}, \\
& \left(\beta_{a, b^{+}}(T)\right)=\Delta_{1}(T)\left(T_{a+b^{+}} T_{-a+b^{+}}\right)\left(T_{a^{+}+b^{+}} T_{-a^{+}+b^{+}}\right)^{-1} \\
& =\left(T_{a+b^{+}} T_{-a^{+}+b^{+}}\right)\left(\alpha+, b^{+}(T)\right), \\
& \left(\gamma_{a, c}(T)\right)=\Delta_{2}(T)\left(T_{a+b} T_{-a+b} T_{b}\right)\left(T_{a+b}^{3}\right)^{-1}, \\
& \left(a^{+}, b^{+} \in \mathbf{Z}(3)^{+r} ; a, b \in \mathbf{Z}(3)^{r}\right) .
\end{aligned}
$$

We shall first show some typical relations between

$$
X_{3, a}(Q \mid U)=\sum_{m \in Z^{r}} Q\left(m+\frac{a}{3} \cdot m+\frac{a}{3}\right)^{3} U\left(m+\frac{a}{3}\right)^{6} \quad\left(a \in \mathbf{Z}(3)^{r}\right)
$$

with coefficients in $R(r, 3)=\mathrm{Z}\left[\left(T_{a}\right)_{a \in Z(3)^{r}}\right]$ :

\section{Proposition 2.}

$$
\begin{aligned}
& \Delta_{1}(T) X_{3}, a+b(Q \mid U) X_{3},-a+b(Q \mid U) \\
& =\sum_{0^{+} \in Z(3)+r} \beta_{a, c}(T) X_{\mathbf{3}}, c^{++b}(Q \mid U) X_{\mathbf{3},-c^{++b}}(Q \mid U), \\
& \Delta_{2}(T) X_{3}, a+b(Q \mid U) X_{3,-a+b}(Q \mid U) X_{3}, b(Q \mid U) \\
& =\sum_{c \in Z(3)^{r}} r_{a}, c(T) X_{3 \cdot c+b}(Q \mid U)^{3}, \quad\left(a, b \in \mathbf{Z}(3)^{r}\right) \text {. }
\end{aligned}
$$

Proof. As we shall see in $\S 3$ the determinants $\Delta_{1}(T)$ and $\Delta_{2}(T)$ are not zero. By virtue of (14) it follows 


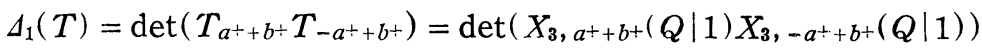

$$
\begin{aligned}
& =\operatorname{det}\left(Q\left(\frac{b^{+}}{3}, \frac{b^{+}}{3}\right)^{6} X_{3}, a^{+}\left(Q \mid Q\left(\frac{b^{+}}{3}\right)\right) X_{3},-a^{+}\left(Q \mid Q\left(\frac{b^{+}}{3}\right)\right)\right) \\
& =\prod_{b^{+}}\left(Q ( \frac { b ^ { + } } { 3 } \cdot \frac { b ^ { + } } { 3 } ) ^ { 6 } \operatorname { d e t } \left(X_{3}, a^{+}\left(Q \mid Q\left(\frac{b^{+}}{3}\right)\right) X_{3},-a^{+}\left(Q \mid Q\left(\frac{b^{+}}{3}\right)\right)\right.\right. \\
& \neq 0 \text {, } \\
& \Delta_{2}(T)=\operatorname{det}\left(T_{a+b}^{3}\right)=\operatorname{det}\left(X_{3}, a+b(Q \mid 1)^{3}\right) \\
& =\operatorname{det}\left(Q\left(\frac{b}{3}, \frac{b}{3}\right)^{9} X_{3}, a\left(Q \mid Q\left(\frac{b}{3}\right)\right)^{3}\right) \\
& =\prod_{b}\left(Q\left(\frac{b}{3}, \frac{b}{3}\right)^{9} \operatorname{det}\left(X_{3}, a\left(Q \mid Q\left(\frac{b}{3}\right)\right)^{3}\right)\right. \\
& \neq 0 \text {. }
\end{aligned}
$$

Since $R(r, 3)$ is an integral domain, it follows that $\left\{X_{3}, c(Q \mid U) X_{3},-c(Q \mid U) c^{+} \in\right.$ $\left.\mathbf{Z}(3)^{+r}\right\}$ and $\left\{X_{3}, a(Q \mid U)^{3} \mid a \in \mathbf{Z}(3)^{r}\right\}$ are sets of linearly independent formal theta functions of level 6 and 9, respectively. By virtue of (15) formal theta functions of level 6 (resp. level 9) $\sum_{m \in \mathbf{Z}} \lambda_{m} U(m)^{2}$ such that $\lambda_{3 m+1}=\lambda_{3 m-1}=0\left(m \in \mathbf{Z}^{r}\right)$ form a vector space of dimension 6 (resp. dimension 9) over the quotient field of $R(r, 3)$. Therefore we may put

$$
\begin{aligned}
& X_{3}, a(Q \mid U) X_{3,-a}(Q \mid U)=\sum_{c^{+} \in Z^{(3)+r}} \lambda_{a, c} X_{3, c^{+}}(Q \mid U) X_{3,-c^{+}}(Q \mid U), \\
& X_{3, a}(Q \mid U) X_{3,-a}(Q \mid U) X_{3,0}(Q \mid U)=\sum_{c \in Z(3) r} \mu_{a, c} X_{3, c}(Q \mid U)^{3} .
\end{aligned}
$$

Putting $U=Q\left(\frac{c}{3}\right)\left(c \in \mathbf{Z}(3)^{r}\right)$ we have

$$
\lambda_{a, c}=\Delta_{1}(T)^{-1} \beta_{a, c}(T), \mu_{a, c}=\Delta_{2}(T)^{-1} \gamma_{a, c}(T),\left(a, c \in \mathbf{Z}(3)^{r}, c^{+} \in \mathbf{Z}(3)^{+r}\right) .
$$

For every $b \in \mathbf{Z}(3)^{r}$ by virtue of (15) it follows

$$
\begin{aligned}
& \Delta_{1}(T) X_{3, a+b}(Q \mid U) X_{3,-a+b}(Q \mid U)=\sum_{c^{+} \in \mathbf{Z}(3)+r} \beta_{a, c^{+}}(T) X_{3, c^{+}+b}(Q \mid U) X_{3,-c^{++b}}(Q \mid U), \\
& \Delta_{2}(T) X_{3, a+b}(Q \mid U) X_{3,-a+b}(Q \mid U) X_{3, b}(Q \mid U)=\sum_{c \in Z(3) r} \gamma a, c(T) X_{3, c+b}(Q \mid U)^{3} .
\end{aligned}
$$

\section{$\S 3$. The explicite defining relations of abelian schemes of level three}

3.1. Let $\left(Y_{a}\right)_{a \in \mathbf{Z}(3)^{r}}$ be a system of indeterminates and $R(r, 3)[Y]$ be the graded ring $R(r, 3)\left[\left(Y_{a}\right)_{a \in \mathbf{Z}(3)}{ }^{r}\right]$. Let $I_{v}$ be the homogeneous ideal in $R(r, 3)[Y]$ generated by the following homogeneous elements

$$
\begin{array}{r}
\left\{k_{l, a}(T) Y_{a+b} Y_{-a+b}-\sum_{\left.c^{+} \in \mathbf{Z}^{(3)}\right)^{r}} h_{3, a} c^{+}(T) Y_{c^{++b}} Y_{-c^{++b}} \mid h_{3, a, c^{+}}(T) h_{l, a}(T)^{-1}\right. \\
\left.=\beta_{a, c^{+}}(T) \Delta_{1}(T)^{-1} ; a, b \in \mathbf{Z}(3)^{r}, c^{+} \in \mathbf{Z}(3)^{+r}\right\}
\end{array}
$$


and

$$
\begin{gathered}
\left\{h_{2, a}(T) Y_{a+b} Y_{-a+b} Y_{b}-\sum_{c \in Z(3)^{r}} h_{4, a, c}(T) Y_{c+b}^{3} \mid h_{4, a, c}(T) h_{2, a}(T)^{-1}\right. \\
\left.=\gamma_{a, c}(T) \Delta_{2}(T)^{-1} ; a, b, c \in \mathbf{Z}(3)^{r}\right\} .
\end{gathered}
$$

Since $\Delta_{1}(T), \Delta_{2}(T), \beta_{a, c^{+}}(T), \gamma_{a, c}(T)$ are homogeneous elements in $R(r, 3)$ such that $\operatorname{deg} \Delta_{1}(T)=\operatorname{deg} \beta_{a, c^{+}}=2^{r}$ and $\operatorname{deg} \Delta_{2}(T)=\operatorname{deg} \gamma_{a, c}(T)$ the homogeneous ideal $I_{v}$ of $R(r, 3)[Y]$ induces an indeal $\Im_{v}$ of the $O_{S(r, 3)}$-algebra $O_{S(r, 3)}[Y]$. We denote by $V(r, 3)$ the $S(r, 3)$-projective scheme $\operatorname{Proj}_{S(r, 3)}\left(O_{S(r, 3)}[Y] / \mathcal{G}_{V}\right)$ of the graded $O_{S(r, 3)}$ algebra $O_{S(r, 3)}[Y] / \Im_{v}$.

We mean by $\left(X_{a}\right)_{a \in \mathbf{Z}_{(3)}}{ }^{r}$ the image of $\left(Y_{a}\right)_{a \in \mathbf{Z}(3)^{r}}$ in the residue ring $R(r, 3)[Y] / I_{V}$ and by $R[X]$ the graded ring $R(r, 3)\left[\left(X_{a}\right)_{a \in \mathbf{Z}_{(3)} r}\right]$. The elements $X_{a}\left(a \in \mathbf{Z}(3)^{r}\right)$ are characterized by the relations:

$$
\begin{aligned}
& h_{1, a}(T) X_{a+b} X_{-a+b}=\sum_{\left.0^{+} \in \mathbf{Z}^{(3)}\right)^{+r}} h_{3, a, c^{+}}(T) X_{c^{+}+b} X_{-c^{+}+b}, \\
& \left(h_{3, a, c^{+}}(T) h_{1, a}(T)^{-1}=\beta_{a, c^{+}}(T) \Delta_{1}(T)^{-1}, a, b \in \mathbf{Z}(3)^{r}, c^{+} \in \mathbf{Z}(3)^{+r}\right) \\
& h_{2, a}(T) X_{a+b} X_{-a+b} X_{b}=\sum_{\left.c \in \mathbf{Z}^{(3)}\right)^{r}} h_{4, a, c}(T) X_{c+b}^{3}, \\
& \qquad\left(h_{4, a, c}(T) h_{2, a}(T)^{-1}=\gamma a, c(T) \Delta_{2}(T)^{-1}, a, b, c \in \mathbf{Z}(3)^{r}\right) .
\end{aligned}
$$

By virtue of Proposition 2 the formal theta functions $X_{3, a}(Q \mid U)\left(a \in \mathbf{Z}(3)^{r}\right)$ satisfy the relations (20) and (21). Hence the map: $X_{a} \rightarrow X_{3, a}(Q \mid U)\left(a \in \mathbf{Z}(3)^{r}\right)$ may be extended to an $O_{S(r, 3)}$-morphism $\rho$ of $O_{S(r, 3)}[Y] / \Im_{r}$ onto $O_{S(r, 3)}\left[\left(X_{3, a}\right.\right.$ $\left.(Q \mid U)_{a \in \mathbf{Z}(3)}\right]$. The dual $\rho^{*}$ of $\rho$ gives the injection morphism of $A(r, 3)$ into $V(r, 3)$.

3.2. When $r=1$, the relation (21) is reduced to a single relation

$$
\left(T_{0}^{3}+2 T_{1}^{3}\right) X_{0} X_{1} X_{-1}=T_{0} T_{1}^{2}\left(X_{0}^{3}+X_{1}^{3}+X_{-1}^{3}\right)
$$

and the relation (20) is trivial. We shall express $\Delta_{1}(T), \Delta_{2}(T), \beta_{a, c^{+}}(T), \gamma_{a, c}(T)$ :

$$
\begin{aligned}
& \Delta_{1}(T)=\operatorname{det}\left(\begin{array}{c}
T_{0} T_{0}, T_{1} T_{1} \\
T_{1} T, T_{-1} T_{0}
\end{array}\right)=T_{1}\left(T_{0}^{3}-T_{1}^{3}\right) \neq 0 \\
& \Delta_{2}(T)=\operatorname{det}\left(\begin{array}{c}
T_{0}^{3}, T_{1}^{3}, T_{-1}^{3} \\
T_{1}^{3}, T_{-1}^{3}, T_{0}^{3} \\
T_{-1}^{3}, T_{0}^{3}, T_{1}^{3}
\end{array}\right)=3 T_{0}^{3} T_{1}^{6}-\left(T_{0}^{9}+2 T_{1}^{9}\right) \neq 0 \\
& \beta_{01}(T)=\beta_{ \pm 0,1}(T)=0, \beta_{00}(T)=\beta_{ \pm 1,1}(T)=\Delta_{1}(T) \\
& \gamma_{0, \pm 1}(T)=0, \gamma_{0,0}(T)=\Delta_{2}(T), \gamma_{ \pm 1,0}(T)=\gamma_{ \pm 1,1}=\gamma_{ \pm 1,-1} .
\end{aligned}
$$

We denote by $T_{a}^{(i)}$ the power series 


$$
T_{a}^{(i)}=X_{a}^{(i)}\left(Q_{i i} \mid 1\right) \sum_{m \in \mathbf{Z}(3)} Q_{i i}^{3(m+a / 3)^{2}}, \quad(1 \leq i \leq r ; a \in \mathbf{Z}(3)),
$$

and denote by $X_{a}^{(i)}(1 \leq i \leq r ; a \in(3))$ the quantities defined by the relation:

$$
\left(T_{0}^{(i) 3}+2 T_{1}^{(i) 2}\right) X_{0}^{(i)} X_{1}^{(i)} X_{-1}^{(i)}=T_{0}^{(i)} T_{1}^{(i) 2}\left(X_{0}^{(i) 3}+X_{1}^{(i) 3}+X_{-1}^{(i) 3}\right)
$$

$(1 \leq i \leq r)$.

We denote by $R_{i}^{*}(1,3)$ the subring $Z\left[\left(T_{a}^{(i)}\right)_{a \in(3)}\right]_{\left(\Delta_{1}\left(T^{(i)}\right) \Delta_{2}\left(T^{(i)}\right)\right)}$ of degree zero in the quotient ring of $Z\left[\left(T_{a}^{(i)}\right)_{a \in \mathbf{Z}_{(3)}}\right]$ with respect to $\Delta_{1}\left(T^{(i)}\right) \Delta_{2}\left(T^{(i)}\right),(1 \leq i \leq r)$ and by $R^{*}(r, 3)$ the subring $R_{\left(\Delta_{1}(T) \Delta_{2}(T)\right)}$ of degree zero in the quotient ring of $R$ with respect to $\Delta_{1}(T) \Delta_{2}(T)$. We denote by $S^{*}(1,3)$ and $S^{*}(r, 3)$ the affine scheme $\operatorname{Spec}\left(R_{1}^{*}(1,3)\right)$ and $\operatorname{Spec}\left(R^{*}(r, 3)\right)$, respectively

Since $Q_{i i}(1 \leq i \leq r)$ are indetermenates and $T_{a}^{(i)}=T_{3, a}^{(i)}(Q)(a \in \mathbf{Z}(3))$ are formal power series in $Q_{i i}$, the map:

$$
T_{a 1}^{(1)} \otimes \cdots \otimes T_{a r}^{(r)} \rightarrow T_{a 1}^{(1)} \cdots T_{a r}^{(r)} \quad\left(a_{1}, \ldots, a_{r} \in \mathbf{Z}(3)\right)
$$

induces an isomorphism of $R_{1}^{*}(1,3) \otimes \cdots \otimes R_{r}^{*}(1,3)$ onto the subring $R^{* *}$ of degree zero in the quotient ring of $\mathbf{Z}\left[\left(T_{a_{1}}^{(1)} \cdots T_{a_{r}}^{(r)}\right)_{a_{1}}, \ldots, a_{r} \in \mathbf{Z}_{(3)}\right]$ with respect to $\prod_{i=1}^{r} \Delta_{1}\left(T^{(i)}\right) \Delta_{2}\left(T^{(i)}\right)$. For the sake of simplicity we shall identify $T_{a_{1}}^{(1)} \otimes \cdots \otimes$ $T_{a_{r}}^{(r)}$ with $T_{a 1}^{(1)} \cdots T_{a_{r}}^{(r)},\left(a, \ldots, a_{r} \in \mathbf{Z}(3)\right)$. Let $F(Y)$ be an element in $\mathbf{Z}[Y]$. Then $F(T)=0$ means that $F(T(Q))$ is zero as a formal rational power series. Therefore an equality $F(T)=0$ implies $F\left(T^{(1)} \otimes \cdots \otimes T^{(r)}\right)=0$, because, replacing $Q_{i, j}(i \neq j)$ by 1 in $F(T(Q))$, we have $F\left(T^{(1)} \otimes \cdots \otimes T^{(r)}\right)$. This shows that the map:

$$
\left.T_{\left(a_{1}\right.}, \ldots, a_{r}\right) \rightarrow T_{a_{1}}^{(1)} \otimes \cdots \otimes T_{a_{r}}^{(r)}=T_{a_{1}}^{(1)} \cdots T_{a_{r}}^{(r)}, \quad\left(a_{1}, \ldots, a_{r} \in \mathbf{Z}(3)\right)
$$

induces a surjective morphism $\lambda: R^{*}(r, 3) \rightarrow R^{* *}=R_{1}^{*}(1,3) \otimes \cdots \otimes R_{r}^{*}(1.3)$.

The dual $\lambda^{*}$ of $\lambda$ is the injection morphism:

$$
S^{* *}=S^{*}(1,3) \times \stackrel{r}{r} \times S^{*}(1,3) \rightarrow S^{*}(r, 3),
$$

where $S^{* *}$ is considered as the affine $\operatorname{scheme} \operatorname{Spec}\left(R^{* *}\right)$.

We mean by $I^{* *}$ the homogeneus ideal in $R^{* *}[Y]$ generated by

$$
\begin{aligned}
& \lambda\left(\Delta_{1}(T)\right) Y_{a+b} Y_{-a+b}-\sum_{0^{+} \in \mathbf{Z}(3)^{+r}} \lambda\left(\beta_{a, c^{+}}(T)\right) Y_{c^{++b}} Y_{-c^{++b}}, \\
& \lambda\left(\Delta_{2}(T)\right) Y_{a+b} Y_{-a+b} Y_{b}-\sum_{c \in \mathbf{Z}(3)^{r}} \lambda\left(\gamma_{a, c}(T)\right) Y_{c+b}^{3}, \quad\left(a, b \in \mathbf{Z}(3)^{r}\right) .
\end{aligned}
$$

We mean by $\left(Z_{a}\right)_{a \in \mathbf{Z}_{(3)^{r}}}$ the image of $\left(Y_{a}\right)_{a \in \mathbf{Z}_{(3)^{r}}}$ in the residue ring $R^{* *}[Y] / I^{* *}$. Then $\left(Z_{a}\right)_{a \in \mathbf{Z}(3)^{r}}$ satisfies the relations: 


$$
\begin{aligned}
& Z_{a+b} Z_{-a+b}=\left(\Delta_{1}(T)\right)^{-1} \sum_{c^{+} \in \mathbf{Z}(3)^{r}} \lambda\left(\beta_{a, c^{+}}(T)\right) Z_{c^{+}+b} Z_{-c^{++}}, \\
& Z_{a+b} Z_{-a+b} Z_{b}=\lambda\left(\Delta_{2}(T)\right)^{-1} \sum_{c \in \mathbf{Z}(3)^{r}} \lambda(\gamma a, c(T)) Z_{c+b}^{3}, \quad\left(a, b \in \mathbf{Z}(\mathbf{3})^{r}\right) .
\end{aligned}
$$

We shall prove the isomorphism:

$$
(V(1,3) \times \cdots \times V(1,3)) \times_{s(1,3) \times \cdots \times s(1,3)} S^{* *}=V(r, 3) \times_{s(r, 3)} S^{* *} .
$$

\section{Lemma 1.}

$$
\begin{aligned}
& \lambda\left(\Delta_{1}(T)\right)=\Delta_{1}\left(T^{(1)}\right) \cdots \Delta_{1}\left(T^{(r)}\right), \\
& \lambda\left(\Delta_{\varepsilon}(T)\right)=\Delta_{2}\left(T^{(1)}\right) \cdots \Delta_{2}\left(T^{(r)}\right) \text {, }
\end{aligned}
$$

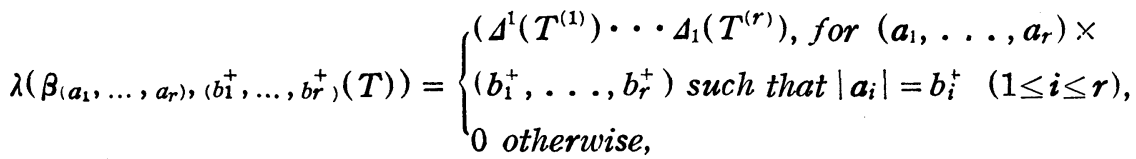

$$
\begin{aligned}
& \lambda\left(\gamma_{\left(a_{1}, \ldots, a_{r}\right),\left(b_{1}, \ldots, b_{r}\right)}(T)\right)=\gamma_{a_{1}, b_{1}}\left(T^{(1)}\right) \cdots \gamma_{r, b_{r}}\left(T^{(r)}\right), \\
& \left(a_{1}, \ldots, a_{r}, b_{1}, \ldots, b_{r} \in \mathbf{Z}(3)\right) \text {, }
\end{aligned}
$$

where we mean

$$
|a|= \begin{cases}a & \text { for } a=0,1 \\ -a & \text { for } a=-1\end{cases}
$$

Proof. From the definition it follows:

$$
\begin{aligned}
& \lambda\left(\Delta_{i}(T)\right)=\Delta_{i}(\lambda(T))=\Delta_{i}\left(T^{(1)} \otimes \cdots \otimes T^{(r)}\right) \\
& =\Delta_{i}\left(T^{(1)}\right) \otimes \cdots \otimes \Delta_{i}\left(T^{(r)}\right)=\Delta_{i}\left(T^{(1)}\right) \cdots \Delta_{i}\left(T^{(r)}\right),
\end{aligned}
$$

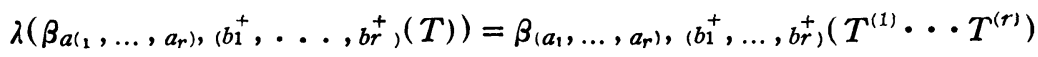

$$
\begin{aligned}
& =\beta_{a_{1}}, b_{1}^{+}\left(T^{(1)}\right) \cdots \beta_{a_{r}}, b_{r}^{+}\left(T^{(r)}\right) \\
& =\left\{\begin{array}{l}
\left.\Delta_{1}(T)^{(1)}\right) \cdots \Delta_{1}\left(T^{(r)}\right), \text { for }\left(a_{1}, \ldots, a_{r}\right) \times \\
\left(b_{1}^{+}, \ldots, b_{r}^{+}\right) \text {such that }\left|a_{i}\right|=b_{i}^{+}(1 \leq i \leq r), \\
0 \text { otherwise, }
\end{array}\right. \\
& \lambda\left(\gamma_{\left(a_{1}, \ldots, a_{r}\right),\left(b_{1}, \ldots, b_{r}\right)}(T)\right)=\gamma_{\left(a_{1}, \ldots, a_{r}\right),\left(b_{1}, \ldots, b_{r}\right)}\left(T^{(1)} \cdots T^{(r)}\right) \\
& =\gamma_{a_{1}, b_{r}}\left(T^{(1)}\right) \cdots \gamma_{a_{r}, b_{r}}\left(T^{(r)}\right) \text {, } \\
& \left(a_{1}, \ldots, a_{r}, b_{1}, \ldots, b_{r} \in \mathbf{Z}(3)\right) .
\end{aligned}
$$

Leмma 2. It follows the relations:

$$
\begin{aligned}
& Z_{\left(a_{1}+b_{1}, \ldots, a_{r}+b_{r}\right)} Z_{\left(-a_{1}+b_{1}, \ldots,-a_{r}+b_{r}\right)}=Z_{\left(\left|a_{1}\right|+b_{1}, \ldots,\left|a_{r}\right|+b_{r}\right)} Z_{\left(-\left|a_{1}\right|+b_{1}, \ldots,-\left|a_{r}\right|+b_{r}\right)}, \\
& Z_{\left(b_{1}, \ldots, b_{i-1}, a+b_{i}, b_{i}+1, \ldots, b_{r}\right)} Z_{\left(b_{1}, \ldots, b_{i-1}, a+b_{i}, b_{i}+1, \ldots, b_{r}\right)} Z_{\left(b_{1}, \ldots, b_{r}\right)} \\
& \left.=\Delta\left(T^{(i)}\right)^{-1} \sum_{r \in \mathbf{Z}(3)} \gamma, c\left(T^{(i)}\right) Z_{\left(b_{1}, \ldots, b_{i-1}, c+b_{i}, b_{i}+1\right.}, \ldots, b_{r}\right), \\
& \left(1 \leq i \leq r ; a_{1}, \ldots, a_{r}, b_{1}, \ldots, b_{r} \in \mathbf{Z}(3)\right) .
\end{aligned}
$$


This is a consequence of the definition of $\left(Z_{a}\right)_{a \in \mathbf{Z}(3)}$ and Lemma 1 .

Lemma 3. Let $\left(c_{1}, \ldots, c_{r}\right)$ be a fixed element in $\mathrm{Z}(3)^{r}$ and put

$$
Z_{a}^{(i)}=Z_{\left(c_{1} \ldots, c_{i-1}, i, c_{i+2} \ldots, c_{r}\right)} \quad(1 \leq i \leq r ; a \in \mathbf{Z}(3)) .
$$

Then it follows

$$
Z_{(1, \ldots r)} Z_{\left(c_{1}, \ldots, c_{r}\right)}^{r-1}=Z_{a_{1}}^{(1)} \cdots Z_{a_{r}}^{(r)}, \quad\left(a_{1}, \ldots a_{r} \in \mathbf{Z}(3)\right) .
$$

Proof. From Lemma 2 it follows

$$
\begin{aligned}
& Z_{\left(a_{1}, \ldots, a_{r}\right)} Z_{\left(c_{2}, \ldots c_{r}\right)}=Z_{\left(a_{1}, c_{2}, \ldots, c_{r}\right)} Z_{\left(c_{1}, a_{2}, \ldots, a_{r}\right)} \\
& Z_{\left(c_{1}, a_{2}, \ldots, a_{r}\right)} Z_{\left(c_{1}, \ldots c_{r}\right)}=Z_{\left.c_{1}, a_{2}, c_{3}, \ldots, c_{r}\right)} Z_{\left(c_{1}, c_{2}, a_{3}, \ldots, a_{r}\right)}, \\
& \cdot \cdot \cdot \\
& Z_{\left(c_{1}, \ldots, c_{r-2}, a_{1}, a_{2}\right)} Z_{\left(c_{1}, \ldots, c_{r}\right)}=Z_{\left(c_{1}, \ldots, c_{r-2}, a_{r-1}, c_{r}\right)} Z_{\left(c_{1}, \ldots, c_{r-1}, a_{r}\right)}
\end{aligned}
$$

Hence, making the product of the both sides of these equations, we have

$$
Z_{\left(a_{1}, \ldots, a_{r}\right)} Z_{\left(c_{1}, \ldots, c_{r}\right)}=Z_{a_{1}}^{(1)} \cdots Z_{a_{r}}^{(r)}, \quad\left(a_{1}, \ldots, a_{r} \in \mathbf{Z}(3)\right) .
$$

Lemma $4 . \quad(V(1,3) \times \cdots \times V(1,3)) \times_{s(1,3) \times \cdots \times s(1,3)} S^{* *}=V(r, 3) \times_{s(r, 3)} S^{* *}$ (as $S(r, 3)$-scheme).

Proof. We denote by $D_{+}\left(Z_{c}\right)$ the affine open subscheme $\left\{z \in V(r, 3) \times_{s(r, 3)} S^{* *}\right.$ $\left.\mid Z_{c}(z) \neq 0\right\}$ and by $D_{+}\left(X_{c_{1}}^{(1)} \cdots X_{c_{r}}^{(r)}\right)$ the affine open subscheme $\left\{\boldsymbol{u}_{1} \times \cdots \times\right.$ $u_{r} \in(V(1,3) \times \cdots \times V(1,3)) \times s(1,3) \times \cdots \times s(1,3) S^{* *}\left\{X_{c_{1}}^{(1)}\left(u_{1}\right) \cdots X_{c_{r}}^{(r)}\left(u_{r}\right) \neq 0\right\}$, where $\left(X_{a}^{(i)}\right)_{1 \leq i \leq r, a \in Z_{(3)}}$ is a system of quantities defined by

$$
\Delta_{1}\left(T^{(i)}\right) X_{0}^{(i)} X_{1}^{(i)} X_{-1}^{(i)}-\gamma\left(T^{(i)}\right)\left(X_{0}^{(i) 3}+X_{3}^{(i) 3}+X_{-1}^{(i) 3}\right)=0 \quad(1 \leq i \leq r) .
$$

From Lemma 1 it follows

$$
\begin{aligned}
& \lambda\left(\Delta_{1}(T)\right)\left(X_{a_{1}+b_{1}}^{(1)} \otimes \cdots \otimes X_{a_{r}+b_{r}}^{(r)}\right)\left(X_{-a_{1}+b_{1}}^{(1)} \otimes \cdots \otimes X_{-a_{r}+b_{r}}^{(r)}\right) \\
& -\sum_{c_{1}{ }^{+}, \ldots, c_{2}+} \lambda\left(\beta_{\left(a_{1}, \ldots, a_{r}\right),\left(c_{1}{ }^{+}, \ldots c_{1}{ }^{+}, \ldots, c_{1}{ }^{+}\right.}(T)\right)\left(X_{c_{1}+b_{1}}^{(1)} \otimes \cdots \otimes X_{c_{r}+b_{r}}^{(r)}\right) \\
& \left(X_{\left.-c^{+}+b_{1}\right)}^{(1)} \otimes \cdots \otimes X_{-c r^{+}+b_{r}}^{(1)}\right) \\
& =\left[\Delta_{1}\left(T^{(1)}\right) X_{a_{1}+b 1}^{(1)} X_{-a_{1}+b_{1}}^{(1)}-\sum_{c_{1}+} \beta_{a_{1}, c_{1}+}\left(T^{(r)}\right) X_{c_{1}++b 1}^{(1)} X_{-c 1^{+}+b_{1}}^{(1)}\right] \otimes \ldots \otimes \\
& {\left[\Delta_{1}\left(T^{(r)}\right) X_{a_{r}+b_{r}}^{(r)} X_{-a_{r}+b_{r}}^{(r)}-\sum_{c_{r}+} \beta_{a_{r}, c_{r}}+\left(T^{(r)}\right) X_{c^{+}+b_{r}}^{(r)} X_{-c_{r}+}^{(r)}{ }^{+} b_{r}\right]=0,} \\
& \lambda\left(\Delta_{2}(T)\right)\left(X_{a_{1}+b_{1}}^{(1)} \cdots X_{a_{r}+b_{r}}^{(r)}\right)-\sum_{\left(c_{1}, \ldots, c r\right)} \lambda(\gamma(a),(c)(T)) X_{c_{1}+b_{1}}^{(1)} \otimes \cdots \otimes X_{c r+b r}^{(r)} \\
& \left.=\left[\Delta_{2}\left(T^{(1)}\right) X_{a_{1}+b_{1}}^{(1)} X_{-a_{1}+b_{1}}^{(1)} X_{b_{1}}^{(1)}-\sum_{c_{1}} \gamma_{a_{1}, c_{1}}\left(T^{(1)}\right) X_{c_{1}+b_{1}}^{(1) 3}\right)\right] \otimes \cdots \otimes \\
& {\left[\Delta_{2}\left(T^{(r)}\right) X_{a_{r}+b_{r}}^{(r)} X_{-a_{r}+b_{r}}^{(r)} X_{b_{r}}^{(r)}-\sum_{c r} r_{a_{r}, c_{r}}\left(T^{(r)}\right) X_{c_{r}+b_{r}}^{(r) 3}\right]=0 .}
\end{aligned}
$$


This shows that the map: $Z_{\left(a_{1}, \ldots, a_{r}\right)} \rightarrow X_{a_{1}}^{(1)} \cdots X_{a_{r}}^{(r)}\left(a_{1}, \ldots, a_{r} \in Z(3)\right)$ induces the injection morphism $\psi$ of $(V(1,3) \times \cdots \times V(1,3)) \times{ }_{s(1,3) \times \cdots \times s(1,3)} S^{* *}$ into $V(r, 3) \times_{s(r, 3)} S^{* *}$ such that $\psi\left(D_{+}\left(X_{c_{1}}^{(1)} \cdots X_{c_{r}}^{(r)}\right)\right) \subseteq D_{+}\left(Z_{\left(c_{1}, \ldots, c_{r}\right)}\right)$. We shall construct the inverse morphism of $\phi$. Put $Z_{a}^{(i)}=Z_{\left(c_{1}, \ldots, c_{i-1}, a, c_{i+1}, \ldots, c_{r}\right)}(1 \leq i \leq r$; $a \in(3))$. Then by virtue of Lemma 1 and 2 it follows

$$
\begin{aligned}
& \quad Z_{a+b}^{(i)} Z_{-a+b}^{(i)}-Z_{|a|+b}^{(i)} Z_{-|a|+b}^{(i)} \\
& \quad=Z_{\left.1 c_{1}, \ldots, c_{i-1}, a+b, c_{i+1}, \ldots, c_{r}\right)} Z_{\left(c_{1}, \ldots, c_{i-1},-a \pm b, c_{i+1}, \ldots, c_{r}\right)} \\
& \quad-Z_{\left(c_{1}, \ldots, c_{i-1},|a|+b, c_{i+1}, \ldots, c_{r}\right)} Z_{\left(c 1, \ldots, c_{i-1},-|a|+b, c_{i+1}, \ldots, c_{r}\right)}=0, \\
& Z_{a+b}^{(i)} Z_{-a+b}^{(i)} Z_{b}^{(i)}-\Delta_{2}\left(T^{(i)}\right)^{-1} \sum_{c} \gamma_{a, c}\left(T^{(i)}\right) Z_{c+b}^{(i) 3} \\
& \quad=Z_{\left(c_{1}, \ldots, c_{2-1}, a+b, c_{i+1}, \ldots, c_{r}\right)} Z_{\left(c_{1}, \ldots, c_{i-1},-a+b, c_{i+1}, \ldots, c_{r}\right)} Z_{\left.c_{1}, \ldots, c_{i-1}, b_{i+1}, \ldots, c_{r}\right)} \\
& \quad-\Delta_{2}\left(T^{(1)}\right) \cdots \Delta_{2}\left(T^{(r)}\right) \sum \gamma_{\left(c_{1}, \ldots, c_{i-1}, a, c_{i+1} \cdots c_{r}\right),\left(c_{1}, \ldots, c_{i-1}, d, c_{i+1}, \ldots, c_{r}\right)} \\
& \left(T^{(1)} \oplus \cdots \otimes T^{(r)}\right) Z^{3}{ }_{\left(c_{1}, \ldots, c_{i-1}, b+d, c_{i+1}, \ldots, c_{r}\right)}=0 .
\end{aligned}
$$

Therefore by virtue of Lemma 3 it follows that the map:

$$
X_{a_{1}}^{(\mathrm{r})} \cdots X_{a_{r}}^{(r)} \rightarrow Z_{a_{1}}^{(1)} \cdots Z_{a_{r}}^{(r)}=Z_{\left(1, \ldots, a_{r}\right)} Z_{\left(c_{1}, \ldots, a_{r}\right)}^{-1}\left(a_{1}, \ldots, a_{r} \in \mathbf{Z}(3)^{r}\right)
$$

induces the injective morphism $\psi_{\left(c_{1}, \ldots, c_{r}\right)}^{\prime}$ of $D_{+}\left(Z_{\left(c_{1}, \ldots, c_{r}\right)}\right)$ into $D_{+}\left(X_{c_{1}}^{(1)}, \ldots\right.$, $\left.X_{c_{r}}^{(1)}\right)$. These morphisms $\psi$ and $\phi^{\prime}\left(c, \ldots, c_{r}\right)$ are the inverse each other as $S(r, 3)$ morphisms between $D_{+}\left(X_{c_{1}}^{(1)} \cdots X_{c_{r}}^{(1)}\right)$ and $D\left(Z_{\left(c_{1}, \ldots, c_{r}\right)}\right)$. Since $\psi$ is defined on $(V(1,3) \times \cdots \times V(1,3)) \times{ }_{s(1,3) \times \cdots \times s(1,3)} S^{* *}$, there exists an $S(r, 3)$-morphism $\psi^{\prime}$ such that $\phi^{\prime} \mid D\left(Z_{\left(c_{i}, \ldots, c_{r}\right)}\right)=\psi_{\left(c_{1}, \ldots, c_{r}\right)}^{\prime}$. This completes the proof of Lemma 4 .

3.3. We denote by $M_{R^{*}(r, 3)}(Y, m)$ the $R^{*}(r, 3)$-submodule in $R^{*}(r, 3)[Y]$ consisting of all the elements of degree $m$, by $M_{R^{*}(r, 3)}(X, m)$ the $R^{*}(r, 3)$-submodule in $R^{*}(r, 3)[X]$ consisting of all the elements of degree $m$ and by $I_{R(r, 3)}(m)$ the $R^{*}(r, 3)$-submodule in the kernel of $R^{*}(r, 3)[Y]$ onto $R^{*}(r, 3)[X]$ consisting of all the elements of degree $m$. For a point $x$ in $S=S(r, 3)$ we mean by $M_{O_{s, x}}(X, m), M_{c_{s, x}}(Y, m), I_{o_{s}, x}(m), M_{k_{x}}(Y, m), M_{k_{x}}(Y, m), I_{k_{x}}(m)$ the tensor products

$$
\begin{aligned}
& M_{R^{*}(r, 3)}(X, m) \otimes_{R^{*}(r, 3)} O_{S, x}, \quad M_{R^{*}(r, 3 j}(X, m) \otimes_{R^{*}(r, 3)} O_{S, x}, \quad I_{R(r, 3)} \otimes_{R^{*}(r, 3)} O_{S, x}, \\
& M_{O_{S, x}}(Y, m) \otimes o_{S, x} k_{x}, \quad M_{O_{S, x}}(X, m) \otimes_{o_{S}, k_{x}}, \quad I_{O_{S, x}}(m) \otimes_{o_{S, x}} k_{x},
\end{aligned}
$$

respectively. Then it follows the exact sequence

$$
\begin{aligned}
& 0 \rightarrow I_{R(r, 3)}(m) \rightarrow M_{R^{*}(r, 3)}(Y, m) \rightarrow M_{R^{*}(r, 3)}(Y, m) \rightarrow 0 \\
& 0 \rightarrow I_{O_{S, x}}(m) \rightarrow M_{O_{S}, x}(Y, m) \rightarrow M_{O_{S}, x}(X, m) \rightarrow 0 \\
& I_{k_{x}} \rightarrow M_{k_{x}}(Y, m) \rightarrow M_{k_{w}}(X, m) \rightarrow 0
\end{aligned}
$$


Lemma 4. Let $x_{0}$ be the generic point on $S(r, 3)$ and $y$ be any point on $S^{*}$ $S^{*}(r, 3)$. Then it follons:

$$
\operatorname{rank}_{k_{x_{0}}} M_{k_{x_{0}}}(X, m) \leqq \operatorname{rank}_{k_{y}} M_{k_{y}}(X, m) \quad(m=1,2,3, \ldots) .
$$

Proof. Since $O_{s, x_{0}}$ is the quotient field of $R^{*}(r, 3)$, it follows $k_{x_{0}}=O_{s, x_{0}}$ and the exact sequence

$$
0 \rightarrow I_{k_{x_{0}}}(m) \rightarrow M_{k_{x_{0}}}(Y, m) \rightarrow M_{k_{x_{0}}}(X, m) \rightarrow 0 .
$$

Since $Y_{a}\left(a \in \mathbf{Z}(3)^{r}\right)$ are indeterminates, it follows

$$
\operatorname{rank}_{k_{y}} M_{k_{0}}(Y, m)=\operatorname{rank}_{k_{x_{0}}} M_{k_{x_{0}}}(Y, m) .
$$

Then it is sufficient to prove the inequality

$$
\operatorname{rank}_{k_{x_{0}}} I_{k_{x_{0}}}(m) \geq \operatorname{rank} I_{k_{y}}(m) \quad(m=1,2,3, \ldots) .
$$

Let $L_{1}, L_{2}, \ldots, L_{N(m)}$ be all the monomials of degree $m$ in $Y_{a}\left(a \in \mathbf{Z}(3)^{r}\right)$. Then there exists a matrix with coefficients in $R^{*}(r, 3)$ :

$$
\Omega^{(m)}=\left(\omega_{i, j}^{(m)}\right), \quad(1 \leq i \leq \lambda(m), 1 \leq j \leq N(m))
$$

such that

$$
\sum_{j=1}^{N(m)} \omega_{i, j}^{(m)} L_{j} \quad(1 \leq i \leq \lambda(m))
$$

generates $I_{R^{*}(r, 3)}(m)$. Let $\mathfrak{p}_{y}$ be the prime ideal in $R^{*}(r, 3)$ corresponding to a point $y$ in $S^{*}(r, 3)$. Then it follows

$$
\begin{aligned}
& \operatorname{rank}_{k_{x_{0}}} I_{k_{x_{0}}}(m)=\operatorname{rank}_{k_{x_{0}} \Omega^{(m)}, \operatorname{rank}_{k_{y}} I_{k_{y}}(m)=\operatorname{rank}_{k_{y}}\left(\Omega^{(m)} \bmod g_{y}\right),} \\
& \qquad(m=1,2,3, \ldots) .
\end{aligned}
$$

This implies

$$
\operatorname{rank}_{k_{0}} I_{k_{0}}(m) \geq \operatorname{rank}_{k_{y}} I_{k_{y}}(m), \quad(m=1,2,3, \ldots) .
$$

Proposition 3. Let $x_{0}$ be the generic point on $S(r, 3)$. Then it follows

$$
V(r, 3) \times{ }_{s(r, 3)} x_{0} \simeq A(r, 3) \times s(r, 3) x_{0} .
$$

Proof. Let $z_{0}^{(1)}$ be the generic point in $S(1,3)$. Then $V(1,3) \times_{S(1,3)} z_{0}^{(1)}$ is defined by the equation $\left(T_{0}^{3}+2 T_{1}^{3}\right) X_{0} X_{1} X_{-1}-\left(T_{0} T_{1}^{2}\right)\left(X_{0}^{3}+X_{1}^{3}+X_{-1}^{3}\right)=0$. On the other hand the scheme $A(1,3) \times_{s(1,3)} z_{0}^{(1)}$ is also defined by a cubic equation. This shows that Proposition 3 is true for $r=1$. Let $z_{0}$ be the generic point in $S^{* *}$ $=S^{*}(1,3) \times \overbrace{\cdots \cdots \times}^{r} S^{*}(1,3)$. Then by virtue of Lemma 3 we have 


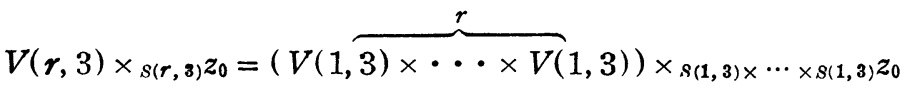

$$
\begin{aligned}
& =(V(1,3) \overbrace{\left.\times_{s(1,3)} z_{0}^{(11)}\right) \times \cdots \times(V}^{r}(1,3) \times \times_{s(1,3)} z_{0}^{(1)}) \\
& =(A(1,3) \overbrace{\left.\times_{s(1,3)} z_{0}^{(1)}\right) \times \cdots \times(A}^{r}(1,3) \times{ }_{s(1,3 ;} z_{0}^{(1)}) \\
& =(A(1,3) \times \overbrace{\cdots \cdots \times A(1,3)) \times}^{r}{ }_{s(1,3) \times \cdots \times s(1,3)}, z_{0} \\
& =(A(1,3) \times \cdots \times A(1,3)) \times_{s(r, 3)} z_{0} \\
& =A(r, 3) \times{ }_{s(r, 3)} z_{0} .
\end{aligned}
$$

From (22) it follows

$$
\operatorname{rank}_{k_{z_{0}}(1)} M_{k_{z_{0}}(1)}\left(X^{(1)}, m\right)=3 m, \quad(m=1,2,3, \ldots) .
$$

From the above relation we have

$$
\operatorname{rank}_{k_{z_{0}}} M_{k_{z_{0}}}(X, m)=3^{r} m^{r}, \quad(m=1,2,3, \ldots) .
$$

Therefore from Lemma 4 it follows

$$
\operatorname{rank}_{k_{x_{0}}} M_{k_{x_{0}}}(X, m) \leq \operatorname{rank}_{k_{z_{0}}} M_{k_{z_{0}}}(X, m) \leq 3^{r} m^{r}, \quad(m=1,2,3, \ldots) .
$$

On the other hand by virtue of Proposition 2 there exists an injection $\rho^{*}$ of $A(r, 3)$ into $V(r, 3)$ as $S(r, 3)$-scheme. From Proposition 1 this implies

$$
\operatorname{rank}_{k_{x_{0}}} M_{k_{x_{0}}}(X, m) \geq 3^{r} m^{r}, \quad(m=1,2,3, \ldots) .
$$

Hence we have the relations

$$
\begin{array}{r}
\operatorname{rank}_{k_{x_{0}}} M_{k_{x_{0}}}(X, m)=3^{r} m^{r}, \operatorname{rank}_{k_{x_{0}}} I_{k_{x_{0}}}(X, m)=N(m)-3^{r} m^{r} \\
(m=1,2,3, \ldots) .
\end{array}
$$

Let $J_{k_{0}}$ be the ideal in $k_{x_{0}}[Y]$ corresponding to the closed scheme $\rho^{*}(A(r, 3))$ $\times_{s(r, 3)} x_{0}$ and $J_{k_{0}}(m)$ the $k_{x_{0}}$-submodule in $J_{k_{0}}$ consisting of all the elements of degree $m$. Then by virtue of Proposition 1 and 2 it follows

$$
\begin{aligned}
\operatorname{rank} J_{k_{x_{0}}}(m)=N(m)-3^{r} m^{r}, \operatorname{rank} J_{k_{x_{0}}}(m) \leq \operatorname{rank} I_{k_{x_{0}}}(m), \\
\quad(m=1,2,3, \ldots) .
\end{aligned}
$$

This implies $J_{k_{x_{0}}}=I_{k_{x_{0}}}$. Namely we have the isomorphism between scheme $V(r, 3) \times s(r, 3) x_{0}$ and $A(r, 3) \times s(r, 3) x_{0}$.

Finally we shall state the main theorem:

Theorem 1. There exists an open subscheme $U(r, 3)$ in $S(r, 3)$ such that the $U(r, 3)$-scheme 


$$
V(r, 3) \times_{s(r, 3)} U(r, 3)
$$

is an abelian scheme, and that $U(r, 3) \times \mathrm{z} G F(p)$ is a non-empty open set in $S(r, 3) \times \mathrm{z} G F(p)$ for every finite prime $p^{4)}$.

\section{§4. The explicite addition formula for theta functions of level theree}

4.1. The addition formula for theta functions of level three is simple and beautiful. First we shall introduce theta functions in $U \otimes_{R} \cdots \otimes_{R} U$, where $R=R(r, 3)$. We mean by a theta function of level $n$ in $U \otimes_{R} \cdots \otimes_{R} U$ with period $Q \otimes_{R} \cdots \otimes Q_{R}$ a power series

$$
\varphi\left(U \otimes_{R} \cdots \otimes_{R} U\right)=\sum_{m_{1}, \ldots, m_{l} \in \mathbf{Z}^{r}} \lambda_{\left(m_{1}, \ldots, m_{l}\right)} U\left(m_{1}\right)^{2} \otimes_{R} \cdots \otimes_{R} U\left(m_{e}\right)^{2}
$$

such that

$$
\begin{aligned}
& \left.\varphi\left(Q\left(m_{1}\right) \otimes_{R} \cdots \otimes_{R} Q\left(m_{l}\right)\right)\left(U \otimes_{R} \cdots \otimes_{R} U\right)\right) \\
& =Q\left(m_{1}, m_{1}\right)^{-n} \cdots Q\left(m_{l}, m_{l}\right)^{-n}\left(U\left(m_{1}\right)^{-2 n} \otimes \cdots \otimes U\left(m_{l}\right)^{-2 n}\right)\left(U \otimes_{R} \cdots \otimes_{R} U\right), \\
& \quad\left(m_{1}, \ldots, m_{l} \in \mathbf{Z}^{\mathbf{r}}\right) .
\end{aligned}
$$

Then, similarly as Proposition $l$, the tensor products $X_{3, a_{1}}(Q \mid U) \otimes_{R} \cdots \otimes_{R} X_{3, a_{l}}$ $(Q \mid U)\left(a_{1}, \ldots, a_{\iota} \in \mathbf{Z}(3)^{r}\right)$ form a base of theta functions of level three in $U \otimes_{R} \cdots \otimes_{R} U$ over the quotient field of $R$. Similarly as Propositinn 2 the tensor products $\left(X_{3, c^{++a}}(Q \mid U) X_{3,-c^{++a}}(Q \mid U)\right) \otimes_{R}\left(X_{3, d^{++b}}(Q \mid U) X_{3,-d^{++b}}(Q \mid U)\right)$ $\left(c^{+}, d^{+} \in \mathbf{Z}(3)^{+r}, a, b \in \mathbf{Z}(3)^{r}\right)$ form a base of theta functions of level six in $U \otimes{ }_{R} U$.

We mean by $X_{3, a}\left(Q \mid U \otimes{ }_{R} U\right)$ and $X_{3, a}\left(Q \mid U \otimes U^{-1}\right)$ the power series

$$
\sum_{m \exists \mathbf{Z}^{r}} Q\left(m+\frac{a}{3}, m+\frac{a}{3}\right)^{3} U\left(m+\frac{a}{3}\right)^{6} \otimes{ }_{R} U\left(m+\frac{a}{n}\right)^{6}
$$

and

$$
\sum_{m \in \mathbf{Z}^{r}} Q\left(m+\frac{a}{3} \cdot m+\frac{a}{3}\right)^{3} U\left(m+\frac{a}{n}\right)^{6} \otimes{ }_{R} U\left(m+\frac{a}{n}\right)^{-6}, \quad\left(a \in \mathbf{Z}(3)^{r}\right) .
$$

In these notation the addition formula is expressed as follows:

\section{THEOREM 2. (The addition formula)}

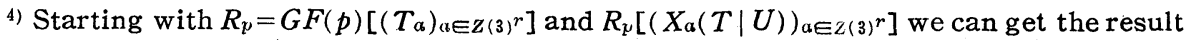
over $G F(p)$ similar as that over $\mathbf{Z}$ in $\$ 3$ (by the same method). This shows that $U(r, 3)$ $\times{ }_{z} G F(p)$ is a non-empty open set. 


$$
\begin{aligned}
& \Delta_{1}(T) X_{3, a+b}\left(Q \mid U \otimes{ }_{R} U\right) X_{3, a-b}\left(Q \mid U \otimes{ }_{R} U^{-1}\right) \\
& \quad=\sum_{c^{+}, a^{+} \in \mathbf{Z}(3)+r} \alpha_{c^{+}, d^{+}}(T)\left(X_{z}, c^{++a}(Q \mid U) X_{3,-c^{+}+a}(Q \mid U)\right) \\
& \otimes_{R}\left(X_{3, d+b}(Q \mid U) X_{3},-d^{++b}(Q \mid U)\right), \quad\left(a, b \in \mathbf{Z}(3)^{r}\right) .
\end{aligned}
$$

Proof. From the definitions it follows the relations

$$
\begin{aligned}
& X_{3, a+b}\left(Q \mid U \otimes{ }_{R} U\right) X_{3, a-b}\left(Q \mid U \otimes{ }_{R} U^{-1}\right) \\
& =\sum_{m^{\prime}, m^{\prime \prime} \in \mathbb{Z}^{r}} Q\left(m^{\prime}+\frac{a+b}{3}, m^{\prime}+\frac{a+b}{3}\right)^{3} Q\left(m^{\prime \prime}+\frac{a-b}{3}, m^{\prime \prime}+\frac{a-b}{3}\right)^{6} \\
& \left(U\left(m^{\prime}+\frac{a+b}{3}\right)^{3} \otimes{ }_{R} U\left(m^{\prime}+{ }^{a+b}\right)^{6}\right)\left(U\left(m^{\prime \prime}+\frac{a-b}{3}\right)^{6} \otimes{ }_{R} U\left(m^{\prime \prime}+\frac{a-b}{3}\right)^{-6}\right) \\
& =\sum_{m^{\prime}, m^{\prime \prime} \in \mathbf{Z}^{r}} Q\left(m^{\prime}+\frac{a+b}{3}, m^{\prime}+\frac{a+b}{3}\right)^{3} Q\left(m^{\prime \prime}+\frac{a-b}{3}, m^{\prime \prime}+\frac{a-b}{3}\right)^{3} \\
& U\left(3\left(m^{\prime}+m^{\prime \prime}\right)+2 a\right)^{2} \otimes{ }_{R} U\left(3\left(m^{\prime}-m^{\prime \prime}\right)+2 b\right)^{2} .
\end{aligned}
$$

This shows that $X_{3, a+b}\left(Q \mid U \otimes_{R} U\right) X_{3,-a-b}\left(Q \mid U \otimes_{R} U^{-1}\right)$ has an expansion $\sum_{m^{\prime}, m^{\prime} \prime^{\prime}} \lambda_{3 m^{\prime}+2 a, 3 m^{\prime \prime}+2 b} U\left(3 m^{\prime}+2 a\right) \otimes{ }_{R} U\left(3 m^{\prime \prime}+2 b\right)$. On the other hand from the difference equation (10) it follows

$$
\begin{aligned}
& \left.X_{3, a+b}\left(Q \mid Q\left(m^{\prime}\right) \otimes_{R} Q\left(m^{\prime \prime}\right)\right)\left(U \otimes_{R} U\right)\right) X_{3, a_{-} b}\left(Q \mid\left(Q\left(m^{\prime}\right) \otimes_{R} Q\left(m^{\prime \prime}\right)^{-1}\right)\left(U \otimes_{R} U^{-1}\right)\right) \\
& \left.\left.=Q\left(m^{\prime}, m^{\prime}\right)^{-6} Q\left(m^{\prime \prime}, m^{\prime \prime}\right)^{-6} U\left(m^{\prime}\right)^{-12} \otimes_{R} U(m)^{-12} X_{3, a+b}\left(Q \mid U \otimes_{R} U\right) X_{3, a-b}\right) Q \mid U \otimes_{R} U^{-1}\right) .
\end{aligned}
$$

Therefore we have

$$
\begin{aligned}
& X_{3, a+b}\left(Q \mid U \otimes{ }_{R} U\right) X_{3, a-b}\left(Q \mid U \otimes{ }_{R} U^{-1}\right) \\
& =\sum_{c^{+}, d^{+} \in Z_{(3)+r}+{ }^{+}, d^{+}}\left(X_{3, c^{++}}(Q \mid U) X_{3,-c^{++a}}(Q \mid U)\right) \otimes_{R}\left(X_{3, d^{++b}}(Q \mid U) X_{3,-d^{++}}(Q \mid U)\right) .
\end{aligned}
$$

Putting $U=Q\left(\frac{c}{3}\right)\left(c \in \mathbf{Z}(3)^{r}\right)$, we have

$$
\lambda_{c^{+}, d^{+}}=\Delta_{1}(T)^{-1} \alpha_{c^{+}, d^{+}}(T), \quad\left(c^{+}, d^{+} \in \mathbf{Z}(3)^{+r}\right) .
$$

\section{Reference}

[1] H. Morikawa, On theta functions and abelian varieties over valuation fields of rank one, I. Nagoya Math. Jour. Vol. 20 (1962).

[2] $\mathrm{H}$. Morikawa, On theta functions and abelian varieties over valuation fields of rank one II. Nagoya Math. Jour. Vol. 21 (1962).

\section{Mathematical Institute}

Nagoya University 